\title{
Experiência da residência multiprofissional em serviço social e cuidados paliativos oncológicos
}

\author{
Andréa Geórgia de Souza Frossard \\ Instituto Nacional de Câncer José Alencar Gomes da Silva \\ (INCA)
}

Emanuel Cristiano de Souza Silva

Serviço Social do Comércio Departamento Regional Ceará (SESC-CE)

Experiência da residência multiprofissional em Serviço Social e cuidados paliativos oncológicos Resumo: Este artigo destaca a inserção do programa de residência multiprofissional no Serviço de Terapia da Dor e Cuidados Paliativos do Instituto do Câncer do Ceará (ICC) entre os anos de 2013 a 2015. Apropriando-se da teoria crítica marxiana, apresenta um estudo descritivo e exploratório, de cunho qualitativo, iluminando a dimensão técnico-operativa do Serviço Social no âmbito do ICC. Destacase a importância da dimensão formativa articulada à investigativa, dando centralidade aos processos pedagógicos criativos e adequados ao modelo de residência em curso no país e sua importância na área de Cuidados Paliativos.

Palavras-chave: Residência multiprofissional. Cuidados paliativos. Serviço Social.

The Experience of a Multi-professional Residence in Social Service and Oncological Palliative Care Abstract: This article highlights the insertion of the multi-professional residence program in the Pain Therapy and Palliative Care Service of the Cancer Institute of Ceará (ICC) between 2012 and 2015. Using critical Marxian thinking, it presents a descriptive and exploratory study, of a qualitative nature, to illuminate the technical-operative dimension of social service in the realm of the ICC. It highlights the importance of the educational dimension in relation to the investigative dimension, focusing on the creative pedagogical processes suitable to the residence model practiced in Brazil and their importance in the field of palliative care.

Keywords: Multi-professional residence. Palliative Care. Social Service. 


\section{Introdução}

Na prática cotidiana novos desafios se apresentam ao assistente social impostos pela dinâmica da sociedade, sendo necessária a articulação da dimensão técnico-operativa ao projeto ético-político da profissão. A dimensão formativa está articulada entre a dimensão interventiva e a dimensão investigativa. Em unidades hospitalares, espaço histórico de inserção, situações de sofrimento e perda ocasionados com a morte ou proximidade da finitude humana confrontam o assistente social cotidianamente (GUERRA, 2007). Nestas instituições, as respostas a estas demandas se organizam em processos de trabalho multiprofissionais que, em geral, apontam para uma perspectiva interdisciplinar, de forma que o assistente social, assim como outros profissionais de saúde, tem nas questões práticas entraves e possibilidades na construção de um fazer coletivo o núcleo comum de ação (MERHY, 2002).

As particularidades da questão social influenciam o adoecimento e o tratamento oncológico. A atenção ao paciente, familiares e cuidadores demanda conhecimentos específicos ao profissional de Serviço Social. Neste cenário de prática é imprescindível a divulgação dos direitos sociais, especialmente os que se especificam aos pacientes oncológicos, elaborando estratégias para ampliação e controle social das políticas públicas. Os Programas de Residência Multiprofissional pertencem às estratégias de qualificação do assistente social neste âmbito. No intento de estruturar a interface entre educação e saúde no contexto da educação especializada, os Programas de Residência Multiprofissional em Saúde foram criados com a Lei n. 11.129, de 2005, orientados pelas diretrizes e princípios do Sistema Único de Saúde (SUS), baseados nas necessidades locais e regionais em consonância com o perfil socioepidemiológico da população brasileira.

No Brasil, historicamente a modalidade de ensino denominada residência se consolidou como especialização para médicos vinculada à prática liberal, individual e privatista. A atuação por especialidade é, ainda hoje, característica médica predominante, muitas vezes fragmentando o conhecimento, apartando-o das relações com outros fenômenos e da totalidade que os cerca. Experiências iniciais com a formação de residências abrangendo outras categorias profissionais se deram a partir da década de 1970, mas é apenas com o Movimento de Reforma Sanitária e com a implementação do SUS que se fortalece a demanda por um reordenamento da educação formal na área da saúde (DALLEGRAVE, 2008). A residência, caracterizada como modalidade de formação em serviço, vem ganhando força como estratégia de formação de trabalhadores da saúde, tendo regulamentação capaz de dar amplitude nacional: os programas de residência são financiados pelo Ministério da Saúde e executados em instituições públicas, privadas e filantrópicas. Reconhecendo a importância da ação multidisciplinar no âmbito da saúde, as categorias abrangidas são: biomedicina, ciências biológicas, educação física, enfermagem, fonoaudiologias, medicina veterinária, nutrição, odontologia, psicologia, serviço social e terapia ocupacional (RESOLUÇÃO CNS, 287/1998).

No Ceará, a Residência Multiprofissional em Saúde se apresenta como estratégia de educação permanente na área da cancerologia a partir de 2013. Na perspectiva da integralidade e da atenção em rede, a Escola de Saúde Pública do Ceará (ESP-CE) em parceria com o Instituto do Câncer do Ceará (ICC) encontram-se no processo de consolidação do programa. Este tem por objetivo formar lideranças técnicas, científicas e políticas para qualificar a linha de cuidado à pessoa portadora de câncer visando à promoção, proteção e recuperação da saúde embasada na colaboração interprofissional. O curso desenvolve-se em regime de dedicação exclusiva, compondo a carga horária total de 5.760 horas que se dividem em atividades práticas (80\%) e teóricas (20\%). A duração mínima para conclusão do curso é de dois anos (CEARÁ, 2013). Abrange seis categorias profissionais: enfermagem, farmácia, fisioterapia, nutrição, psicologia e serviço social. Os residentes são dispostos em equipe para a promoção da interdisciplinaridade, percorrendo os cenários de prática da cirurgia oncológica, quimioterapia, radioterapia e cuidados paliativos em períodos determinados. Com a preceptoria, compartilham as atividades buscando a qualificação da atenção dispensada, afetando todos os sujeitos envolvidos no espaço de atuação.

Nesse processo, a apropriação dos cuidados paliativos como disciplina e prática instigou inúmeros questionamentos, sendo nosso objetivo relatar a inserção dos residentes no Serviço de Terapia da Dor e Cuidados Paliativos do ICC, descrevendo como se deu a conformação entre as estratégias de intervenção profissional nos cuidados paliativos oncológicos com a residência. Este relato de experiência justifica sua importância na relevância das questões apresentadas, podendo nortear a aplicação de procedimentos ou avaliação de resultados da intervenção em situações similares, colaborando para a práxis metodológica de trabalho do assistente social em cuidados paliativos.

\section{Metodologia}

Trata-se de um estudo de natureza descritiva e exploratória, baseado na teoria crítica marxiana, do tipo relato de experiência, considerando as vivências dos autores com os cuidados paliativos relacionadas à implan- 
tação da Residência Multiprofissional com ênfase em cancerologia. Como se trata de uma área ainda incipiente na produção de conhecimento neste âmbito, optamos por combinar o estudo exploratório, ressaltando, na coleta de informações, os registros de atividades e a observação participante, com a utilização de princípios técnicos da análise etnográfica que é compreensão de processos contemporâneos manifestos nas relações do cotidiano, pois possibilita experiência em que, ao mesmo tempo, observa e distancia-se do presente contexto (MAGNANI, 2009). As interpretações têm base nas percepções do vivenciado pelos autores no decorrer dos anos de 2013 a 2015, nos espaços e processos ligados à Residência Integrada em Saúde, desenvolvidos pela Escola de Saúde Pública do Ceará (ESP-CE) em parceria o Instituto do Câncer do Ceará (ICC).

\section{Discussão e resultados: oncologia e cuidados paliativos}

O câncer é uma doença crônica e progressiva, capaz de afetar praticamente todas as estruturas corporais, caracterizada por divisões celulares desordenadas que comprometem a morfologia e a funcionalidade celulares. Consequentemente, à medida que essas células invadem o tecido, este também vai perdendo sua função. Muitos são os fatores relacionados à etiologia da doença: componente hereditário, fatores de risco não modificáveis (sexo, idade, raça) e modificáveis (alimentação, sedentarismo, alcoolismo, tabagismo, entre outros). As células cancerígenas se agrupam rapidamente formando um tumor extremamente vascularizado, muitas das vezes, capaz de invadir outros tecidos, processo denominado de metástases (LOPES et al, 2005; TEIXEIRA, 2007).

No Brasil, o câncer, como problema de saúde pública, ganha cada vez mais relevância, tendo em vista que apresenta na atualidade altas taxas de incidência e é a segunda maior causa de morte por doença (SALIMENA, 2013). Para os anos de 2016 e 2017 a estimativa é de 596 mil casos novos de câncer no Brasil (INCA, 2015). O prognóstico dos pacientes oncológicos está associado a diversos fatores tais como: diagnóstico precoce (pacientes com maiores chances de cura poderão ser interditado no difícil acesso aos serviços de saúde) e inadequado desenvolvimento de políticas de caráter promocional e preventivo.

Entende-se que uma apropriada abordagem não pode se restringir ao acesso ao tratamento curativo ou de remissão; é preciso compreender que o câncer, como doença crônica evolutiva, pode ocasionar condições de finitude e morte. Não é incomum o sentimento e materialização de situações de desamparo aos que enfrentam esta difícil trajetória. O Brasil é um país marcado por iniciativas fragmentadas de serviços públicos e privados que não materializam uma consistente política pública de cuidados paliativos (RAMALHO; MARTINS, 2007). Um dado relevante diz respeito à qualidade de morte. O país ocupa a 38a posição no ranking de qualidade de morte entre 40 países, conforme divulgado em 2012 pela Economist Intelligence Unit, empresa de consultoria integrante do grupo da revista The Economist. Este estudo considera como alguns fatores que indicam a qualidade da morte: os cuidados prestados aos pacientes no fim da vida e a quantidade de analgésicos disponíveis para o controle da dor. Evidencia-se, assim, um logo caminho a percorrer para a reversão do quadro explicitado, estando o tratamento ainda focalizado na cura atrelada à cultura de que a morte é algo a ser remediado a todo custo, como de costume nas sociedades ocidentais (THE ECONOMIST INTELLIGENCE UNIT, 2015).

Em 2002, o Ministério da Saúde institui no âmbito da saúde pública, através da Portaria GM/MS n. 1.319/2002, o Programa Nacional de Assistência a Dor e os Cuidados Paliativos, observando-se a expansão dos cuidados paliativos. Contudo, este cenário se apresenta como desafiador, sendo aspectos consideráveis nos limites desta expansão, a centralização dos serviços nos grandes centros urbanos, os conflitos de natureza ética, a pequena ampliação da cobertura domiciliar, a qualificação multiprofissional na área, entre outros (FLORIANI; SCHRAMM, 2007). A conclusão de uma terapia curativa para o câncer não significa o fim de um tratamento ativo, uma vez que os cuidados paliativos podem atuar de maneira complementar. A Organização Mundial de Saúde (OMS, 2002) afirma que:

Cuidados paliativos consistem na assistência promovida por uma equipe multidisciplinar, que objetiva a melhoria da qualidade de vida do paciente e seus familiares, diante de uma doença que ameace a vida, por meio da prevenção e alívio do sofrimento, da identificação precoce, avaliação impecável e tratamento de dor e demais sintomas físicos, sociais, psicológicos e espirituais (ANCP, 2012, p.16).

Em cuidados paliativos o foco da atenção é o paciente e não a doença, preconizando uma atenção individualizada e a comunicação franca com o paciente e sua família, buscando a excelência no controle dos sintomas físicos, psicológicos, sociais, espirituais e a prevenção do sofrimento. A avaliação baseia-se no conjunto dos diversos aspectos inclusos na queixa dolorosa que Cicely Saunders, percussora do moderno movimento hospice, definiu como "dor total" (ANCP, 2008). A partir do início dos anos 1960, Cicely Saunders 
elabora em nível conceitual e de prática clínica o termo "dor total” legando um conceito de permanente interesse à área da saúde em que relaciona a natureza multidimensional da experiência da dor, percebida subjetivamente por cada paciente, incluindo fatores físicos, psicológicos, sociais e espirituais nos seus domínios. A assistência em cuidados paliativos não pode se abster desta visão multidimensional na avaliação e controle da dor do paciente, ganhando a escuta da história de vida importância fundamental para se compreender e intervir no sofrimento de forma multifacetada (CLARK, 2010). Atualmente, ainda que se considere a natureza multidimensional da experiência da dor como fundamental para a filosofia dos cuidados paliativos como disciplina, observa-se que o controle da dor na forma como é operacionalizado continua sendo um desafio, tendo como elemento a ser repensado o canal de comunicação entre equipe, pacientes, familiares e cuidadores, como também políticas para área (CHAN; MEHTA, 2008).

A atenção em cuidados paliativos se apresenta em diferentes modelos de assistência, sendo os mais recorrentes no Brasil a assistência hospitalar e em domicílio. Em alguns casos, o paciente solicita ser tratado em sua própria residência o que, a uma primeira impressão, poderia garantir-lhe conforto longe da rigidez da rotina hospitalar e junto à proteção da família, porém sua situação precária de habitação pode ser um fator obstaculizador, por exemplo. Os cuidados paliativos como disciplina enfatizam a necessidade de um olhar mais complexo quanto aos fenômenos inerentes ao adoecimento, ao processo de finitude e de morte, ultrapassando um enfoque biologicista e tecnicista para a intervenção. Neste contexto, o sofrimento do paciente incorpora diferentes dimensões tais como: física, psicológica, social e espiritual. Desta forma, a palavra paliar pressupõe o engendramento de processos de trabalho marcadamente interprofissionais para obtenção da qualidade de vida, no caso de um cenário caracterizado pelas desigualdades sociais e baixo desenvolvimento de políticas neste âmbito (ANCP, 2012). Uma análise crítica rigorosa amplia a compreensão acerca do caráter integrador das refrações da questão social para a obtenção de boas práticas em saúde. Daí a necessária inclusão da família no processo assistencial em curso, bem como, a adequada avaliação socioeconômica e habitacional dos pacientes. A inclusão de uma série de condições objetivas inerentes ao paciente e sua família poderá determinar ou não sua inserção na modalidade de assistência domiciliar (ANCP, 2012).

A natureza complexa e multidimensional do adoecimento em cuidados paliativos está em consonância com a ideia de uma atividade realizada por uma equipe interprofissional em um trabalho convergente no decorrer da doença, na terminalidade da vida, na morte e no período de luto. As diferentes disciplinas buscam de forma intensa a complementaridade do saber e a partilha de responsabilidades, visando ao atendimento às múltiplas necessidades do indivíduo em cuidados paliativos, ultrapassando a característica de agrupamento na busca de engendrar uma equipe integração (PEDUZZI, 2001).

\title{
Serviço Social e cuidados paliativos
}

Na direção da consolidação do trabalho interprofissional nos cuidados paliativos, a atuação do assistente social se dá na interlocução com a instituição contratante, equipe de saúde e usuários, articulando redes de suporte social formais e informais. Frente às necessidades do paciente em fase final de vida, a escuta e acolhida se tornam ferramentas indispensáveis no trabalho, visando ao resgate da autonomia do paciente e favorecer ambiente e condições mais dignas, desta forma:

\begin{abstract}
A atuação do assistente social em equipes de atenção paliativa pode ser resumida em: conhecer paciente, família e cuidadores nos aspectos socioeconômicos, visando ao oferecimento de informações e orientações legais, burocráticas e de direitos, imprescindíveis para o bom andamento do cuidado ao paciente, e para a garantia de morte digna. Cabe a esse profissional também avaliar a rede de suporte social dos envolvidos, para junto a estes acioná-la em situações apropriadas; conhecer e estabelecer uma rede intrainstitucional, no intuito de garantir atendimento preciso ao paciente, além de constituir-se como interlocutor entre paciente/família e equipe nas questões relacionadas aos aspectos culturais e sociais que envolvem o cuidado de forma geral (ANCP, 2012, p. 221).
\end{abstract}

Partindo da compreensão do atual contexto e da conformação da Política Nacional de Atenção Oncológica (PNAO) em época de neoliberalismo e desmonte dos direitos sociais, Chaves (2004, p. 183) reflete acerca das propostas institucionais para o cuidado do paciente com câncer avançado, ganhando relevância o papel do cuidador familiar cuja rotina é alterada em função da nova atividade exercida. Desse modo, é recorrente o afastamento do mesmo de sua ocupação laboral, onde enfrenta uma série de novas dificuldades, já que o provimento do cuidado familiar evidencia a desproteção desses sujeitos sob uma perspectiva do direito a ser garantido pelo Estado, de forma que "O cuidado é recebido pela família como algo dado, sem realização de crítica, não questionado pelos 
cuidadores que, apesar de expressarem as suas dificuldades, e o despreparo, além da falta de treinamento para a atividade exercida, não têm outra opção que não seja cuidar do paciente com câncer avançado". Com a desospitalização do paciente, os processos de trabalho desenvolvidos de forma interdisciplinar intervêm para que o cuidador exerça sua atividade de cuidado, buscando diminuir seu esgotamento físico e mental. Desencadeiamse estratégias para reorganização do cotidiano familiar frente a estas necessidades, onde o trabalho do assistente social se destaca. Cabe ressaltar, que a produção do Serviço Social tem apresentado iniciativas criativas com temas que versam sobre a comunicação em saúde, a atuação interprofissional e o trabalho social com famílias em cuidados paliativos. Por fim, em nível de intervenção profissional, apresentam-se documentos que norteiam a atuação: o Manual da Academia Nacional de Cuidados Paliativos (ANCP, 2012) e os Parâmetros para a Atuação dos Assistentes Sociais na Saúde (CFESS, 2010).

\section{Cenário de prática e territorialização}

O Instituto do Câncer do Ceará (ICC), fundado em 1944, é uma entidade filantrópica de direito privado de referência no tratamento, no ensino e na pesquisa em oncologia no Norte e Nordeste do Brasil. Com o crescimento da demanda se inaugurou, em 1999, o Hospital do Câncer; antes a atenção era dada em nível ambulatorial. Em 2011 o Hospital passou a chamar-se Hospital Haroldo Juaçaba. Além da instituição o ICC é mantenedor da Escola Cearense de Oncologia (ECO) e da Casa Vida, casa de apoio para os pacientes. Hoje o Instituto cobre a maior parte das demandas da população cearense acometida por algum tipo de neoplasia. Em 2011, inaugurou-se no ICC do Serviço de Terapia da Dor e Cuidados Paliativos (STDCP), que incluiu o Programa de Atendimento Domiciliar (PAD) e atendimento em nível ambulatorial especializado na dor oncológica, a Clínica da Dor. O PAD tem por objetivo prestar assistência domiciliar e interdisciplinar qualificada aos pacientes oncológicos da rede SUS em fase avançada do câncer, sem possibilidade de tratamentos curativos e que estão impossibilitados de se locomoverem para acompanhamentos ambulatoriais ou hospitalares. Este programa se destina apenas a usuários do SUS. No STDCP são realizadas atividades científicas como: aulas semanais com temas relativos aos cuidados paliativos, discussões de casos clínicos para os profissionais da equipe e oficinas mensais de educação continuada para pacientes e familiares. O Serviço é coparticipante do $4^{\circ}$ ano de residência médica em anestesiologia da Universidade Federal do Ceará (UFC), na subespecialização de Terapia da Dor e Cuidados Paliativos. A partir do ano de 2013, agregou-se ao Serviço a Residência Multiprofissional em Saúde, recebendo residentes de enfermagem, fisioterapia, nutrição, psicologia e serviço social, que passaram a participar das atividades científicas e assistenciais, sobretudo das visitas domiciliares, objetivando a requalificação da assistência e desenvolvimento do processo formativo. Este Serviço se configurou como um dos cenários de prática onde se deram as atividades da Residência para conhecimento da rede de atenção oncológica. Como proposta inicial de trabalho, no primeiro mês de inserção os residentes deveriam territorializar o contexto da instituição levando em consideração quatro macro-dimensões: organização do serviço, relações de poder, determinantes sociais e comunidades. Tal perspectiva de territorialização proporcionou a apropriação do cenário de trabalho pelos profissionais para conhecer dados socioepidemiológicos, processos de saúde-doença-cuidado, história da instituição, fluxo de organização, dinâmica, limites, potencialidades, modos de vida e trabalho.

No STDCP a atividade se desenvolveu através de rodas de equipe, observações, entrevistas, visitas, capacitações e pesquisa documental. Os resultados foram sistematizados, gerando um relatório final de territorialização e matrizes de análise institucional elencando oportunidades, fragilidades e ameaças. A territorialização, neste âmbito, auxiliou na formação dos profissionais em relação a organização dos cuidados paliativos no ICC e sua inserção na rede e na sociedade. Possibilitou a apropriação adequada do cenário institucional, auxiliando no desenvolvimento de intervenções mais coerentes com o contexto percebido, além de inovar, visto que este procedimento é realizado na atenção em saúde com ênfase comunitária, não sendo comum sua realização em instituições de atenção terciária em saúde.

\section{Processos pedagógicos}

O período vivenciado pelo residente de serviço social nos cuidados paliativos tinha como propósito capacitá-lo através do desenvolvimento das competências necessárias à atuação qualificada neste campo. O residente desenvolveu a capacidade de dar respostas as demandas que se colocam à profissão, apropriando-se do real oriundo do confronto entre a realidade e a possibilidade do fazer profissional. O processo de aprendizado se deu com o suporte da preceptoria que, já familiarizada ao serviço, auxiliou o residente neste percurso 
através da troca de saberes. Os métodos da roda e da tenda invertida foram utilizados para gerir o processo educativo e fomentar as práticas em saúde. As rodas foram realizadas dentro da proposta pedagógica do programa incluindo a roda de campo e a roda de núcleo. Estes momentos se destacaram como espaço dialógico de troca de conhecimento construindo um saber crítico e estimulando o desenvolvimento das competências e tecnologias em cuidados paliativos. De uma forma geral, os diversos temas discutidos nas rodas inerentes à formação em saúde e gestão do trabalho em cuidados paliativos ganharam substância na discussão das questões práticas e situações limites vivenciadas pelos sujeitos envolvidos. Através da construção do conhecimento podemos visualizar elementos de campo e elementos de núcleo para estruturar o trabalho de equipe, realizando práticas adequadas à natureza multifatorial dos fenômenos que demandavam nossa atenção. Estes momentos permitiram a construção de uma visão pautada na integralidade e humanização do cuidado.

Expandindo o ensino ao território, a tenda invertida se firmou como o momento onde o preceptor se deslocou ao território do residente ajudando-o a construir e a refletir sobre suas práticas, sendo a principal metodologia utilizada pelos docentes no processo de formação, transformando o espaço que originalmente era da assistência em local de construção de saberes e práticas. Nestes momentos de troca, o residente se apropriou dos instrumentos utilizados pelo Serviço Social do ICC no STDCP, direcionados à investigação social e utilizando-se tanto de métodos qualitativos como quantitativos, gerando conhecimento que embasou novas respostas às demandas da categoria, além de vários impasses cotidianos.

Considerando o percurso histórico dos cuidados paliativos no Brasil, é necessário o aprimoramento do trabalho em rede. $\mathrm{O}$ mapeamento de recursos sistemáticos mostrou se ser uma boa prática, como o exemplo de programas de residência no Rio de Janeiro. Ainda que os cuidados paliativos sejam campo de trabalho emergente, a rede de fluxos e contrafluxos expôs a dificuldade da materialização da garantia de direitos e de acesso dos usuários às diversas redes

Sistematizar as experiências vividas na prática cotidiana dos serviços é tão importante quanto planejar as atividades, configurando-se como um dos principais instrumentos para refletir sobre o trabalho desenvolvido, conformando uma prática realmente científica e capaz de organizar processos de trabalho articulados à dimensão formativa no Serviço Social. de proteção e garantias sociais, impondo novos desafios aos assistentes sociais em um cenário de crise que ameaça a própria existência do Sistema Único de Saúde. Os Cuidados Paliativos diminuem os custos dos serviços de saúde, além de beneficiarem os pacientes e seus familiares.

\section{Atuação interprofissional}

Os cuidados paliativos como modelo assistencial é indissociável da prática interprofissional, considerando o reconhecimento da natureza multidimensional do sofrimento vivenciado pelo paciente, por familiares e cuidadores. Nos processos de trabalho nos quais se inseriram os residentes, não havia trabalho isolado, o paciente era responsabilidade de todos, cada um atuando em conformidade com suas atribuições e competências, construindo abordagens conjuntas como reuniões com familiares e encaminhamentos, abrindo o caminho para a transdisciplinaridade e interprofissionalidade. Após o recebimento do encaminhamento, era agendada uma primeira visita ao paciente, a visita de inclusão, em que se analisava o paciente a fim de preencher ou não os critérios para inclusão no Programa, tendo como instrumentais técnicos a Palliative Performs Scale realizada pelo médico e pela Avaliação Social do assistente social. Posteriormente, ocorriam visitas subsequentes onde eram reconhecidas novas demandas dos pacientes que eram alvos de intervenções. Em caso de hospitalização para o controle de sintoma, a visita também era realizada. A alta do paciente do programa podia dar-se por meio da melhoria do estado clínico ou do óbito. Em alguns casos, o atendimento se estendia ao pósóbito por questões relacionadas à elaboração do luto.

Neste cenário, pode-se perceber que o assistente social contribui com a decodificação da realidade social do paciente frente à equipe potencializando a atuação conjunta. Muitas vezes, o entrave à intervenção específica de uma categoria relacionava-se aos problemas que seriam alvo da atuação de outro profissional. 
Tais abordagens se delinearam na relação entre os núcleos específicos e os núcleos de ação comum. Entre os procedimentos interprofissionais realizamos: visitas domiciliares, construção da história de vida do paciente, escuta qualificada do familiar ou cuidador, orientação quanto à normas e serviços disponibilizados pelo programa. Usou-se uma abordagem interdisciplinar para acessar necessidades clínicas e psicossociais dos pacientes e suas famílias, incluindo aconselhamento e suporte ao luto. Realizava-se também o estudo de caso interprofissional semanalmente. Detínhamo-nos na construção do projeto terapêutico adequado à singularidade de cada caso; a materialização das ações era restringida pelos limites do Programa e da rede assistencial. Cada profissional contribuía na construção da unicidade do caso, visto que os olhares sobre a mesma realidade nem sempre eram concordantes. Em alguns momentos foi necessário o esforço para conformação de uma plataforma de atuação única e manutenção do vínculo existente entre os membros da equipe.

\section{Desafios e possibilidades}

Alguns desafios à implantação do projeto pedagógico e político da residência: excessiva carga horária a que eram submetidos os residentes, número insuficiente de profissionais para acompanhar o andamento das atividades do residente no cotidiano, deficiências na rede de saúde e serviços que repercutiam no processo formativo, atual contexto político das residências multiprofissionais, e dificuldades de consolidação de um espaço democrático de discussão entre os residentes coordenadores/gestores do Programa.

A proposta da residência, ainda que com dificuldades, sobressaiu-se ao modelo de formação tradicional para profissionais da saúde. Os processos de trabalhos e o território de prática foram valorizados como espaço de aprendizagem direcionados a superação da dicotomia entre teoria e prática em organicidade aos princípios do SUS e do trabalho em cuidados paliativos. A concepção pedagógica pautou-se na educação permanente como princípio pedagógico e metodológico. No trabalho multiprofissional, como princípio operador, sendo a integralidade o princípio referencial, o que em muito se afina aos princípios dos cuidados paliativos.

\section{Considerações finais}

Sistematizar as experiências vividas na prática cotidiana dos serviços é tão importante quanto planejar as atividades, configurando-se como um dos principais instrumentos para refletir sobre o trabalho desenvolvido, conformando uma prática realmente científica e capaz de organizar processos de trabalho articulados à dimensão formativa no serviço social.

O registro de impressões e de estratégias que funcionaram ou que não funcionaram durante a implantação da Residência Multiprofissional em Saúde, permitiu pensar sobre o que já foi realizado e sobre o que se pode aperfeiçoar. A Residência, como modelo formativo de destaque na educação continuada para área de saúde, tem sido inovadora como proposta na área de cuidados paliativos, articulando de forma particular diversos elementos que demarcaram o espaço do serviço social na atuação multiprofissional.

\section{Referências}

ACADEMIA NACIONAL DE CUIDADOS PALIATIVOS (ANCP). Manual de Cuidados Paliativos. Diagraphic, Rio de Janeiro, 2008.

Manual de Cuidados Paliativos. ampl. E atual. - Porto Alegre: Sulina, 2012.

BRASIL. Lei nº 11.129, de 30 de junho de 2005. Disponível em: http//www.planalto.gov.br/ccivil_03/_Ato2004-2006/2005/Lei/L11129.htm. Acesso em: 08 de jul. 2016

. Resolução $n^{\circ}$ 287/98, de 08 de outubro de 1998. Disponível em: http://www.conselho.saude.gov.br/resoluções/1998/Reso287.doc. Acesso em: 28 ago. 2014.

CEARÁ, Governo do Estado. Manual das Residências Integradas em Saúde: 2013 - 2015. Escola de Saúde Pública do Ceará, 2013. CHAN L.S.; MEHTA, A.. Understanding of the Concept of "Total Pain": A Prerequisite for Pain Control. Journal of hospice and palliative nursing. 2008; 10(1): pp.26-32.

CHAVES, A. R.M. O cuidador no provimento do cuidado ao paciente com câncer avançado no Instituto Nacional de Câncer. 2004. 220p. Dissertação (Mestrado em Serviço Social). Programa de Pós-Graduação em Serviço Social. Universidade do Estado do Rio de Janeiro - RJ. CLARK, D. Total pain: the work of Cicely Saunders and the hospice movement. American HYPERLINK “http://eprints.gla.ac.uk/view/ journal_volume/American_Pain_Society_Bulletin.html”PainHYPERLINK “http://eprints.gla.ac.uk/view/journal_volume/ American_Pain_Society_Bulletin.html” HYPERLINK “http://eprints.gla.ac.uk/view/journal_volume/American_Pain_Society_ 
Bulletin.html”SocietyHYPERLINK “http://eprints.gla.ac.uk/view/journal_volume/American_Pain_Society_Bulletin.html”HYPERLINK “http://eprints.gla.ac.uk/view/journal_volume/American_Pain_Society_Bulletin.html"Bulletin.2000; 10(4): pp. 13-15.

CONSELHO FEDERAL DE SERVIÇO SOCIAL (CFESS). Parâmetros para atuação de assistentes sociais na política de saúde. Brasília: CFESS, 2010.

DALLEGRAVE, D. No olho do furacão, na ilha da fantasia: a invenção da Residência Multiprofissional em Saúde. 2008. 91p. Dissertação (Mestrado em Enfermagem). Programa de Pós-Graduação Enfermagem. Universidade Federal do Rio Grande do Sul, Porto alegre-RS.

FLORIANI, C. A.; SCHRAMM, F. R. Desafios morais e operacionais da inclusão dos cuidados paliativos na rede de atenção básica. Cad. Saúde Pública. 2007; 23 (9): pp. 2072-2080.

FROSSARD, A.G.S. Vidas em partidas. Rio de Janeiro: Quartet, 2014.

GUERRA, Y. O Projeto Profissional Crítico: estratégia de enfrentamento das condições contemporâneas da prática profissional. Revista Serviço Social e Sociedade n. 91, São Paulo: Cortez, p. 5-33, 2007.

INSTITUTO NACIONAL DO CÂNCER (INCA). Estimativa do Câncer para o biênio de 2016/2017. Disponível em: http:// www.inca.gov.br/wcm/dncc/2015/estimativa-2016.asp. Acesso em: 10 jan. 2016.

LOPES. A. et al. Oncologia para a graduação. São Paulo: Tecmedd, 2005.

MAGNANI. J.G. C. Etnografia como prática e experiência. Ver. Horizontes antropológicos. 2009. p. 129-156.

MERHY, EE. Saúde: a cartografia do trabalho vivo. São Paulo: HUCITEC; 2002.

ORGANIZAÇÃO MUNDIAL DE SAÚDE (OMS). Cuidados Paliativos. 2002. Disponível em: http://www.who.int/cancer/palliative/ es/. Acesso em: 08 jun. 2016.

PEDUZZI, M. Equipe multiprofissional de saúde: conceito e tipologia. Rev. Saúde Pública, São Paulo. 2001; 35(1): p. $103-109$.

RAMALHO, M.A.N.; NOGUEIRA-MARTINS, M. C. F. Vivências de profissionais de saúde da área de oncologia pediátrica. Revista Psicologia em Estudo. 2007. p. 123-132.

SALIMENA, A. M. O. et al. Estratégias de enfrentamento usadas por enfermeiros ao cuidar de pacientes oncológicos. Revista de Enfermagem da Universidade Federal de Santa Maria, 2013. p. 142-147.

TEIXEIRA, M. Explicação diversa para a origem do câncer, com foco nos cromossomos, e não nos genes, ganha corpo no establishment científico. Revista latino-americana de psicopatologia fundamental, São Paulo. 2007 ;10(4): pp. 664-677.

THE ECONOMIST INTELLIGENCE UNIT. The Quality of dithy: ranking enf-of-life care across the word. Disponível em: http:// graphics.eiu.com/upload/eb/qualityofdeath.pdf. Acesso em: 20 nov. 2015.

\section{Andréa Geórgia de Souza Frossard}

afrossard@inca.gov.br

Doutora em Serviço Social pela Universidade Federal do Rio de Janeiro (UFRJ)

Assistente Social no Instituto Nacional de Câncer José Alencar Gomes da Silva (INCA)

\section{Emanuel Cristiano de Souza Silva}

ecsilva@ sesc-ce.com.br

Graduado em Serviço Social pela Universidade Estadual do Ceará (UECE)

Assistente Social no SESC-CE atuando junto ao setor de Educação em Saúde

\section{INCA}

Rua: Visconde de Santa Isabel, 274, Vila Isabel

Rio de Janeiro - Rio de Janeiro - Brasil

CEP: 20570-120

\section{SESC-CE}

Serviço Social do Comércio- Departamento Regional Ceará

Avenida Duque de Caxias, 1701, Centro

Fortaleza - Ceará - Brasil

CEP: 60035-111 